\title{
SURFACES WITH ISOMETRIC GEODESICS
}

\author{
by C. CHARITOS and P. PAMFILOS
}

(Received 20th September 1989, revised 17th April 1990)

The aim of the paper is to prove the Theorem: Let $M$ be a surface in the euclidean space $E^{3}$ which is
diffeomorphic to the sphere and suppose that all geodesics of $M$ are congruent. Then $M$ is a euclidean sphere.

1980 Mathematics subject classification (1985 Revision): 53, 53A05.

\section{Introduction}

The aim of this paper is to prove the following:

Theorem. Let $M$ be a surface in the euclidean space $E^{3}$, which is diffeomorphic to the sphere $S^{2}$. We suppose that all geodesics of $M$ are congruent. Then $M$ is a euclidean 2-sphere.

The basic idea of the proof is the following: We consider a curve $\Gamma_{0}$ in $E^{3}$ such that each geodesic of $M$ is congruent to $\Gamma_{0}$ and let $k(s)$ be the curvature function of $\Gamma_{0}$. By supposing that $k(s)$ is not constant, we find a surface $S$ in the unit sphere bundle $S^{1}(M)$ of $M$ such that the projection $\pi: S \rightarrow M$ with $\pi\left(v_{p}\right)=p$ is a covering map of $M$. But in this case, an everywhere non-zero vector field, tangent to $M$, can be constructed and it is well-known that this is impossible [5]. So the function $k(s)$ is constant and we get easily that $M$ is a euclidean 2 -sphere.

We would like to make the following remarks:

(i) The hypothesis that $M$ is diffeomorphic to $S^{2}$ is not an essential restriction. In fact, if $M$ is compact and $\pi_{1}(M) \neq 0$, then there are geodesics on $M$ which do not have the same length $[1,2]$. On the contrary, there exist surfaces in $E^{3}$, diffeomorphic to the sphere $S^{2}$, which have all their geodesics closed and of the same length, i.e. the Zoll's surfaces [1].

(ii) Actually, in the above mentioned theorem we may assume that the curve $\Gamma_{0}$ is closed. In fact a theorem of Lusternik guarantees the existence of closed geodesics in $M$ [4]. Lusternik's theorem however, is quite technical and our proof does not depend on any detailed information about $\Gamma_{0}$.

(iii) This theorem would be trivial if we had supposed that there is a fixed point $p$ of $M$, such that for every two geodesics $\gamma_{1}, \gamma_{2}$ passing through $p$ there exists a rotation of $E^{3}$ around $p$ taking $\gamma_{1}$ to $\gamma_{2}$. However such a point $p$ does not in general exist. 
We would like to thank the referee for suggesting the use of the formula, appearing in the proof of the Lemma., which also simplifies the proof of Proposition 3.

We denote by $\langle$,$\rangle the usual inner product in E^{3}$ and by $A$ the shape operator of $M$.

Let $v_{p}$ in $S^{1}(M)$. There exists a unique geodesic $\gamma:(-\infty, \infty) \rightarrow M$ such that $\gamma(0)=p$, $\gamma^{\prime}(0)=v_{p}$. We denote by $\kappa\left(v_{p}\right), \tau\left(v_{p}\right)$ the normal curvature and torsion of $\gamma$ at $p$, and we have that:

$$
\kappa\left(v_{p}\right)=\left\langle A v_{p}, v_{p}\right\rangle, \tau\left(v_{p}\right)=\left\langle A v_{p}, J v_{p}\right\rangle
$$

where in general, by $J v_{p}$ we denote the vector that we obtain if we rotate $v_{p}$ in $T_{p} M$ by $\pi / 2$ and such that $\left\{v_{p}, J v_{p}, n_{p}\right\}$ form a positively oriented basis of $E^{3}$.

In what follows we will refer to them as the curvature and torsion of vectors of $S^{1}(M)$.

We consider now a fixed curve $\Gamma_{0}$ in $E^{3}$ such that each geodesic of $M$ is congruent to $\Gamma_{0}$. Below we suppose that the curvature of $\Gamma_{0}$ is not constant and $\Gamma_{0}$ is not a plane curve. In fact in each case we can easily deduce that all points of $M$ are umbilical and consequently $M$ is a sphere. Let $\alpha(s), s \in(-\infty, \infty)$ be a parametrisation by arc-length of $\Gamma_{0}$ and let $k(s), \tau(s)$ be the curvature and torsion functions of $\alpha(s)$ respectively.

\section{The covering space $S$ of $M$}

In this paragraph we will suppose again that the curvature function $k(s)$ is not constant. So we will find a surface $S$ in $S^{1}(M)$ which is a covering space of $M$.

Proposition 2. Let $\kappa: S^{1}(M) \rightarrow R$ be the differentiable function defined by $\kappa\left(v_{p}\right)=$ $\left|\left\langle A v_{p}, v_{p}\right\rangle\right|$ and let $k_{0}$ be a non-critical value of $k(s)$. Then the set $\kappa^{-1}\left(k_{0}\right)$ is a compact surface in $S^{1}(M)$. have

Proof. Let $v_{p}$ in $\kappa^{-1}\left(k_{0}\right)$ and $\gamma$ be the geodesic of $M$ with $\gamma(0)=p, \gamma^{\prime}(0)=v_{p}$. Then we

$$
\kappa_{*, v_{p}}\left(\gamma^{\prime}(0), \gamma^{\prime \prime}(0)\right)=\left.\frac{d}{d \sigma}\right|_{\sigma=0} \kappa\left(\gamma(\sigma), \gamma^{\prime}(\sigma)\right)=\left.\left.\frac{d k}{d s}\right|_{\mathrm{s}(0)} \cdot \frac{d s}{d \sigma}\right|_{0}
$$

But the last product is different from zero: The term $d k /\left.d s\right|_{s(0)}$ is different from zero because $k_{0}$ is a non-critical value of $k(s)$ and the term $d s /\left.d \sigma\right|_{0}=1$ because the function $s(\sigma)$ defines a reparametrization by arc-length of $\Gamma_{0}$.

So the function $\kappa$ is of rank 1 on $\kappa^{-1}\left(k_{0}\right)$ and consequently $\kappa^{-1}\left(k_{0}\right)$ is a surface in $S^{1}(M)$.

Now the covering space of $M$ will be chosen among the components of a surface $\kappa^{-1}\left(k_{0}\right)$, for a suitable non-critical value $k_{0}$ of $k(s)$. 
Lemma. (i) There is a non-critical value $k_{0}$ of $k(s)$ and a component $S$ of $\kappa^{-1}\left(k_{0}\right)$, which contains at least one non-principal vector.

(ii) Every vector $v$ in $S$ is non-principal.

Proof. (i) The fact that the function $k(s)$ is not constant implies that $M$ contains non-umbilical points. Let $p$ be a non-umbilical point of $M$ and let $v_{p}$ be a unit vector of $T_{p} M$ with $\tau\left(v_{p}\right) \neq 0$; in other words $v_{p}$ is a non-principal vector.

is $\kappa\left(v_{p}\right)=k$ and $k$ is a non-critical value of $k(s)$, then, setting $k_{0}=k$, we choose among the components of $\kappa^{-1}\left(k_{0}\right)$ that one which contains the vector $v_{p}$ and we denote it by $S$.

If $\kappa\left(v_{p}\right)=k$ and $k$ is a critical value of $k(s)$, we consider an open neighbourhood $V$ of $v_{p}$ in $T_{P} M$, sufficiently small, such that $v \in V \Rightarrow \tau(v) \neq 0$. Standard calculations show that

$$
\left.\frac{d}{d t}\right|_{t=0} \kappa((v+t J v) /|v+t J v|)=2 \tau(v)
$$

and hence the values $\kappa(v), v \in V$ form an open subset $U$ in the range of the curvature function $k(s)$. Now by Sard's theorem there exists in $U$ a non-critical value $k_{0}$ of $k(s)$ and evidently if we consider the surface $\kappa^{-1}\left(k_{0}\right)$, there exists a component $S$ of $\kappa^{-1}\left(k_{0}\right)$ which contains a unit vector $v$ with $\tau(v) \neq 0$.

(ii) In $R^{2}$ we consider the curve $\beta(s)=(k(s), \tau(s)), s \in(-\infty, \infty)$. Since $k_{0}$ is a non-critical value of $k(s)$ the set of $s$ with $k(s)=k_{0}$ is a discrete subset of $(-\infty, \infty)$. Therefore there exists at most a countable number of points $s_{i}$ in $(-\infty, \infty), i=$ $1,2, \ldots$ such that $k\left(s_{i}\right)=k_{0}$. Hence the set of values $\tau_{i}=\tau\left(s_{i}\right)$ is also countable, and since $S$ is connected it follows that $\tau$ is constant on $S$. Therefore all vectors in $S$ are non-principal.

We can now prove the following proposition:

Proposition 3. Let $\pi: S \rightarrow M$ be the projection on $M$ with $\pi\left(v_{p}\right)=p$. Then the pair $(S, \pi)$ is a covering space of $M$.

Proof. Consider the curve $\delta(t)=\left(v_{p}+t J v_{p}\right) /\left|v_{p}+t J v_{p}\right|$ in $S^{1}(M)$, for $v_{p} \in S$. Then the tangent vector $\delta^{\prime}(0)$ of $\delta$ spans the kernel of the derivative at $v_{p}$ of the canonical projection map from $S^{1}(M)$ to $M$. However this is transverse to the tangent plane $T_{v p} S$ of $S$, since $d /\left.d t\right|_{t=0} \kappa(\delta(t))=2 \tau\left(v_{p}\right) \neq 0$. Therefore the projection $\pi$ has rank 2 everywhere on $S$ and consequently $\pi$ is a local diffeomorphism.

On the other hand $\pi$ is closed, since $S$ is compact. So $\pi$ is onto and $(S, \pi)$ is a covering space of $M$.

Corollary. The curvature function $k(s)$ of $\Gamma_{0}$ is constant.

Proof. $M$ is simply connected and therefore has no non-trivial covering spaces. So the projection $\pi: S \rightarrow M$ is a diffeomorphism. Now if each $p$ in $M$ is mapped to the 
vector $\pi^{-1}(p)$ of $S$, we get a differentiable vector field tangent to $M$, everywhere non-zero, which is impossible [5]. In this way, the hypothesis that $k(s)$ has non-critical points leads to contradiction. So $k(s)$ is a constant function.

\section{Proof of the theorem}

According to the corollary above, $k(s)$ is a constant function. Therefore all the unit tangent vectors of $M$ have the same curvature, which implies that all the points of $M$ are umbilical. So $M$ is a euclidean 2-sphere.

One should notice here that the above proof works equally well when the surface $M$ is embedded into the hyperbolic space $H^{3}$ or into the sphere $S^{3}$ and all geodesics of $M$ are congruent in $H^{3}$ or $S^{3}$ respectively.

Now it is natural to ask how this theorem is generalized in the case where $M$ is an arbitrary submanifold in a euclidean space $E^{n}$. In fact, suppose that $M$ is a submanifold of codimension $m$ in a euclidean space $E^{n}$, with $m<n-1$. If all geodesics of $M$ are congruent, then the question which arises naturally is the following: Is the submanifold $M$ a sphere or a hyperplane in $E^{n}$, if it is compact or non-compact respectively?

This question is the subject of a forthcoming paper.

\section{REFERENCES}

1. A. Besse, Manifolds all of Whose Geodesics are Closed (Springer-Verlag, 1978).

2. M. Freedman, J. Hass and P. Scott, Closed Geodesics on Surfaces. Preprint, Liverpool University, November 1981.

3. W. Kungeberg, Lectures on Closed Geodesics (Springer-Verlag, 1978).

4. L. Lusternik, The Topology of Function Spaces and the Calculus of Variations in the Large, Translations of Math. Monographs, Vol. 16, Providence, R.I., 1966.

5. J. Milnor, Analytic proofs of the hairy ball theorem, Am. Math. Monthly 85 (1978).

University of Crete

Department of Mathematics

IRAKLION P.O. BOX 470

GrEecE 